\title{
Bootstrap Autoregressive Order Selection
}

\author{
Jürgen Franke, Jens-Peter Kreiss and Martin Moser
}

In this paper we deal with the problem of fitting an autoregression of order $p$ to given data coming from a stationary autoregressive process with infinite order. The paper is mainly concerned with the selection of an appropriate order of the autoregressive model. Based on the so-called final prediction error (FPE) a bootstrap order selection can be proposed, because it turns out that one relevant expression occuring in the FPE is ready for the application of the bootstrap principle. Some asymptotic properties of the bootstrap order selection are proved. To carry through the bootstrap procedure an autoregression with increasing but non-stochastic order is fitted to the given data. The paper is concluded by some simulations.

Keywords: Autoregression; bootstrap; final prediction error; order selection.

\section{Introduction}

In this paper we deal with observations $X_{1}, \ldots, X_{n}$ which are realizations of an infinite order autoregressive model $(\mathrm{AR}(\infty)$-model) of the following type

$$
X_{t}=\sum_{\ell=1}^{\infty} a_{\ell} X_{t-\ell}+\varepsilon_{t}, \quad t \in \mathbb{Z}=\{0, \pm 1, \pm 2, \ldots\} .
$$

The process $\left(\varepsilon_{t}: t \in \mathbb{Z}\right)$ consists of independent and identically distributed (i.i.d.) real valued random variables on a probability space $(\Omega, \mathcal{A}, P)$ with (cumulative) distribution function $F$. Furthermore we assume

$$
E \varepsilon_{1}=0, \quad E \varepsilon_{1}^{2}=\sigma^{2} \in(0, \infty) \text { and } E \varepsilon_{1}^{4}<\infty
$$

The parameter $\mathbf{a}=\left(a_{\ell}: \ell \in I N\right), \mathbb{N}=\{1,2, \ldots\}$, is absolutely summable and the generating function $1-\sum_{\ell=1}^{\infty} a_{\ell} z^{\ell}$ has no zeros in the closed complex unit disk. More formally, $\mathbf{j}(\mathbf{a})=\left(1,-a_{1},-a_{2}, \ldots\right) \in \ell^{1}=\left\{\mathbf{b} \in \mathbb{R}^{N_{0}}:\|\mathbf{b}\|_{1}=\sum_{\ell=0}^{\infty}\left|b_{\ell}\right|<\infty\right\}, N_{0}=$ $\mathbb{N} \cup\{0\}$, and $\mathbf{j}(\mathbf{a})$ is invertible in $\ell^{1}$ with respect to the convolution $(\mathbf{b} * \mathbf{c})_{\ell}=\sum_{j=0}^{\ell} b_{j} c_{\ell-j}$. If $\boldsymbol{\alpha}=\mathbf{j}(\mathbf{a})^{-1}$ denotes the inverse of $\mathbf{j}(\mathbf{a})$ with respect to convolution, then the process $\mathbf{X}=\left(X_{t}\right)$ allows the following representation as an infinite order moving average process 
$(\mathrm{MA}(\infty)$-process)

$$
X_{t}=\sum_{\ell=0}^{\infty} \alpha_{\ell} \varepsilon_{t-\ell}, \quad t \in \mathbb{Z},
$$

where $\alpha_{0}=1$ and the coefficients $\alpha_{\ell}, \ell \geq 1$, can be computed recursively from the convolution equation $\sum_{j=0}^{\ell} \alpha_{j} a_{\ell-j}=0$.

The paper is devoted to the problem of fitting an autoregression of order $\mathrm{p}(\mathrm{AR}(\mathrm{p})$ model) to the given set of data $X_{1}, \ldots, X_{n}$. We start with a brief but careful study of the final prediction error (FPE). We obtain that one of two relevant terms is ready for an approximation through the bootstrap principle. In contrast to the construction of usual order selection procedures (e.g. FPE- or AIC-method), which heavily depend on the kind of the involved parameter estimator (mostly the usual Yule-Walker estimator or the closely related least squares (LS) estimator), the bootstrap approximation is open for other parameter estimates. This takes care of the fact that the more precise we can estimate the parameter of an $A R(p)$-approximation to the given data the higher we probably want to choose the definitive order to obtain a more precise fit. In this context we think of Mestimators or ML-estimators for non-normally distributed situations or so-called adaptive procedures.

The bootstrap procedure is based on a preliminary autoregressive approximation with a non-stochastic order $p_{0}(n)$ converging to infinity. The reader who is interested in a more complete theory for the bootstrap procedure in this $\operatorname{AR}(\infty)$-setup is referred to Kreiss (1988, 1997) and Bühlmann (1997).

The paper is concluded by some simulation results. There the properties of the bootstrap version of FPE are compared with the AIC-method.

\section{An Approximation of the Final Prediction Error}

In this section, we derive an approximation of the well-known FPE-criterion function which is of an appropriate form to apply the bootstrap. Here, we sometimes use heuristic arguments just to motivate this approximation. A rigorous formulation of the asymptotic properties of the order selection procedure, based on this approximation, is postponed to Section 5.

The optimal parameter of a fitted autoregression of order $\mathrm{p}$ is defined as

$$
a(p)=\underset{c(p) \in \mathbb{R}^{p}}{\arg \min } E\left(X_{t}-c(p)^{T} X_{t-1}(p)\right)^{2}
$$

where $X_{t-1}(p)=\left(X_{t-1}, \ldots, X_{t-p}\right)^{T}$. If $\gamma_{h}=E X_{t} X_{t+h}, h \in N_{0}$, denote the autocovariances and

$$
\Gamma(p)=\left(\gamma_{|i-j|}: i, j=1, \ldots, p\right) \text { and } \quad \gamma(p)=\left(\gamma_{1}, \ldots, \gamma_{p}\right)^{T}
$$


then $a(p)$ is given by the Yule-Walker equations

$$
a(p)=\Gamma(p)^{-1} \gamma(p)
$$

We note that under our assumptions on the parameter a the $(p \times p)$-matrix $\Gamma(p)$ is always positive definite, $\Gamma(p)$ and $\Gamma(p)^{-1}$ are uniformly bounded in $p \in I N$ with respect to the operator norm $\|B\|:=\sup \left\{\|B x\|_{2}:\|x\|_{2}:=\left(\sum x_{i}^{2}\right)^{\frac{1}{2}}=1\right\}$ and for the autocovariance function $\gamma=\left(\gamma_{h}: h \in N_{0}\right)$ we have $\gamma \in \ell^{1}$.

On the basis of the given observations $X_{1}, \ldots, X_{n}$ suppose that we have an estimator $\hat{a}(p)=\left(\hat{a}_{1}(p), \ldots, \hat{a}_{p}(p)\right)^{T}$ of $a(p)$ for all $p$ up to a maximal order $p(n)$. The FPE idea suggests to choose the order for the definitive autoregressive fit as

$$
P_{0}(n)=\underset{1 \leq p \leq p(n)}{\arg \min } E^{\mathbf{X}} E^{\mathbf{Y}}\left(Y_{t}-\hat{a}(p)^{T} Y_{t-1}(p)\right)^{2},
$$

where $\mathbf{Y}$ is an independent copy of the time series $\mathbf{X}$. As the number of observations $n$ tends to infinity, the maximal order $p(n)$ is also supposed to converge to infinity. Following Shibata (1980) we obtain

$$
\begin{aligned}
P_{0}(n) & =\underset{1 \leq p \leq p(n)}{\arg \min }\left\{\sigma^{2}+\|\mathbf{a}-a(p)\|_{\Gamma}^{2}+E\|\hat{a}(p)-a(p)\|_{\Gamma(p)}^{2}\right\} \\
& =\underset{1 \leq p \leq p(n)}{\arg \min }\left\{E\left(X_{t}-a(p)^{T} X_{t-1}(p)\right)^{2}+E\|\hat{a}(p)-a(p)\|_{\Gamma(p)}^{2}\right\},
\end{aligned}
$$

where $\Gamma=\left(\gamma_{|i-j|}: i, j \in I N\right),\|x\|_{B}^{2}=x^{T} B x$ and, for the sake of simplicity, $a(p)$ also denotes the $\ell^{1}$-vector $\left(a_{1}(p), \ldots, a_{p}(p), 0, \ldots\right)$ filled up with zeros. Note that $P_{0}(n)$ is a deterministic but not computable quantity. Now the idea is to estimate both parts of the FPE. Let us start with the first expectation

$$
E\left(X_{t}-a(p)^{T} X_{t-1}(p)\right)^{2}=\gamma_{0}-2 a(p)^{T} \gamma(p)+\|a(p)\|_{\Gamma(p)}^{2} .
$$

If we denote by $\hat{\gamma}$ any consistent estimator of the autocovariance function $\gamma$ (we will see later that $\hat{\gamma}$ need not be the empirical autocovariances) this expression can be estimated by

$$
\hat{\gamma}_{0}-2 a(p)^{T} \hat{\gamma}(p)+\|a(p)\|_{\hat{\Gamma}_{(p)}}^{2} .
$$

Next we intend to plug in a further estimate, namely estimators $\hat{a}(p)$ for $a(p)$. As the optimal parameters $a(p)$ correspond to the autocovariance function $\gamma$ through the YuleWalker equations (2.1), the same should hold for the estimators $\hat{a}(p)$ and $\hat{\gamma}$ belonging to them, i.e.

$$
\hat{\Gamma}(p) \hat{a}(p)=\hat{\gamma}(p), \quad 1 \leq p \leq p(n) .
$$

Obviously, $\hat{\gamma}$ needs only to be known up to lag $p(n)$. For ease of notation we do not explicitly indicate the dependence of the estimators on the number $n$ of observations.

This second substitution introduces a systematic bias which, using (2.4), may be calculated as

$$
\begin{aligned}
\left(\hat{\gamma}_{0}\right. & \left.-2 a(p)^{T} \hat{\gamma}(p)+\|a(p)\|_{\hat{\Gamma}(p)}^{2}\right)-\left(\hat{\gamma}_{0}-2 \hat{a}(p)^{T} \hat{\gamma}(p)+\|\hat{a}(p)\|_{\hat{\Gamma}(p)}^{2}\right) \\
& =-2(a(p)-\hat{a}(p))^{T} \hat{\gamma}(p)+(a(p)-\hat{a}(p))^{T} \hat{\Gamma}(p)(a(p)+\hat{a}(p)) \\
& =\|a(p)-\hat{a}(p)\|_{\hat{\Gamma}(p)}^{2} .
\end{aligned}
$$


Because of this a reasonable approximation of the argument of $P_{0}(n)$ is given by

$$
\hat{\gamma}_{0}-\hat{a}(p)^{T} \hat{\gamma}(p)+2 \cdot E\|\hat{a}(p)-a(p)\|_{\Gamma(p)}^{2},
$$

where the expectation is ready for an approximation through the bootstrap, which we will discuss in detail in the next but one section. Note that we have so far not made any assumptions on the estimates $\hat{a}(p)$ and $\hat{\gamma}$ except (2.4).

Finally we need a further approximation of the expectation in (2.5) in order to be able to evaluate some asymptotic properties of the bootstrap order selection. To this end observe that

$$
\|\hat{a}(p)-a(p)\|_{\hat{\Gamma}(p)}^{2}=\left\|\hat{\Gamma}(p)^{-1}(\hat{\gamma}(p)-\hat{\Gamma}(p) a(p))\right\|_{\hat{\Gamma}(p)}^{2}=\|\hat{\gamma}(p)-\hat{\Gamma}(p) a(p)\|_{\hat{\Gamma}(p)^{-1}}^{2} .
$$

We will now make use of the following approximation of the argument of $P_{0}(n)$

$$
\hat{\gamma}_{0}-\hat{a}(p)^{T} \hat{\gamma}(p)+2 \cdot E\|\hat{\gamma}(p)-\hat{\Gamma}(p) a(p)\|_{\Gamma(p)^{-1}}^{2}
$$

The construction of a bootstrap version of the following theoretical order selection procedure

$$
P_{1}(n):=\underset{1 \leq p \leq p(n)}{\arg \min }\left\{\hat{\gamma}_{0}-\hat{a}(p)^{T} \hat{\gamma}(p)+2 \cdot S_{n}(p)\right\}
$$

where

$$
\begin{aligned}
S_{n}(p) & =E\|\hat{\gamma}(p)-\hat{\Gamma}(p) a(p)\|_{\Gamma(p)^{-1}}^{2} \\
& =E\|(\hat{\gamma}(p)-\gamma(p))-(\hat{\Gamma}(p)-\Gamma(p)) a(p)\|_{\Gamma(p)^{-1}}^{2}
\end{aligned}
$$

is exactly the goal of the next but one section. Of course $P_{1}(n)$ is closely related to

$$
P_{1}^{\prime}(n):=\underset{1 \leq p \leq p(n)}{\arg \min }\left\{\hat{\gamma}_{0}-\hat{a}(p)^{T} \hat{\gamma}(p)+2 \cdot E\|\hat{a}(p)-a(p)\|_{\Gamma(p)}^{2}\right\}
$$

\section{Estimators of Prediction Coefficients}

In this section we want to present briefly some estimators for the autocovariance function $\gamma$ or the parameter value $a(p)$ of an autoregressive fit of order $p$ which we have in mind. The easiest situation is to use the empirical autocovariances

$$
\tilde{\gamma}_{h}=\frac{1}{n} \sum_{t=1}^{n-h} X_{t} X_{t+h}, \quad h \in \mathbb{N}_{0},
$$

(or, asymptotically equivalent, their centered version $\tilde{\gamma}_{h}^{c}=\frac{1}{n} \sum_{t=1}^{n-h}\left(X_{t}-\frac{1}{n} \sum_{s=1}^{n} X_{s}\right)\left(X_{t+h}-\right.$ $\left.\frac{1}{n} \sum_{s=1}^{n} X_{s}\right)$ ), to which belong the well-known Yule-Walker parameter estimators

$$
\tilde{a}(p)=\tilde{\Gamma}(p)^{-1} \tilde{\gamma}(p)
$$


In this text we always equip empirical autocovariances and the corresponding Yule-Walker parameter estimators with a tilde. In contrast to these estimators we propose the following alternative. Fit in a first step an autoregression of (high) order $p_{M} \geq p(n)$ to the given data and compute M- or ML-parameter estimators, i.e. solutions of

$$
\Psi_{n}\left(c_{1}, \ldots, c_{p_{M}}\right)=\sum_{t=p_{M}+1}^{n} \psi\left(X_{t}-\sum_{\ell=1}^{p_{M}} c_{\ell} X_{t-\ell}\right) X_{t-1}\left(p_{M}\right) \equiv 0,
$$

where $\psi: \mathbb{R} \rightarrow \mathbb{R}$ denotes a suitable score function. We do not intend to discuss at this place the problem of finding solutions of (3.1). If $p_{M}=p_{M}(n)$ converges to infinity with an appropriate rate and if $\psi$ satisfies some regularity conditions it is possible to find a solution of (3.1), denoted by $\hat{a}^{M}=\hat{a}^{M}\left(p_{M}\right)$, which is consistent for a, cf. Kreiss (1988) and Moser (1997). As $\mathbf{j}(\mathbf{a})$ is invertible in $\ell^{1}$ and the set of invertible sequences in $\ell^{1}$ is open in $\ell^{1}$, we may assume that $\mathbf{j}\left(\hat{a}^{M}\right)$ is invertible as well.

Denote the autocorrelation function belonging to an autoregressive process of order $p_{M}$ with parameter $\hat{a}^{M}$ by $\hat{\mathbf{r}}$. As the autocovariance function $\mathbf{r}$ is a continuous function of the parameter a (with respect to $\|\cdot\|_{1}$ ), $\hat{\mathbf{r}}$ will be a consistent estimator for the theoretical autocorrelation function $\mathbf{r}=\frac{1}{\gamma_{0}} \gamma$. The estimate $\hat{\mathbf{r}}$ coincides with the empirical autocorrelation function up to lag $p_{M}$ if and only if $\hat{a}^{M}$ is the Yule-Walker estimate. $\hat{\mathbf{r}}$ may be computed using the MA $(\infty)$-representation of the AR-process with parameter $\hat{a}^{M}$. As only the components of $\hat{\mathbf{r}}$ up to $\operatorname{lag} p(n) \leq p_{M}$ are needed, an easier approach is to solve

$$
\hat{R}\left(p_{M}\right) \hat{a}^{M}=\hat{r}\left(p_{M}\right),
$$

or equivalently

$$
\hat{C}\left(p_{M}-1\right)\left(\begin{array}{c}
\hat{r}_{1} \\
\vdots \\
\hat{r}_{p_{M}-1}
\end{array}\right)=-\left(\begin{array}{c}
\hat{a}_{1}^{M} \\
\vdots \\
\hat{a}_{p_{M}-1}^{M}
\end{array}\right), \quad \hat{r}_{p_{M}}=\hat{a}_{p_{M}}^{M}+\sum_{\ell=1}^{p_{M}-1} \hat{a}_{p_{M}-\ell}^{M} \hat{r}_{\ell},
$$

where $\hat{C}\left(p_{M}-1\right)=\left(\hat{a}_{i+j}^{M}+\hat{a}_{i-j}^{M}: 1 \leq i, j \leq p_{M}-1\right), \hat{a}_{0}^{M}=-1$ and $\hat{a}_{k}^{M}=0$ if $k<0$ or $k>p_{M}$. As $\mathbf{j}\left(\hat{a}^{M}\right)$ is supposed to be invertible in $\ell^{1}$, the matrix $\hat{C}\left(p_{M}-1\right)$ will be invertible, too.

Based on the autocorrelation estimates $\hat{\mathbf{r}}$, we may calculate new estimates of $a(p)$ using the Yule-Walker equations:

$$
\hat{a}(p)=\hat{R}(p)^{-1} \hat{r}(p), \quad p=1,2, \ldots, p(n) .
$$

Why do we introduce such estimators? It is known that M-estimators $\hat{a}^{M}$ are more efficient for $\mathbf{a}$ if the innovations $\varepsilon_{t}$ are not normally distributed, see e.g. Martin (1983) or Kreiss (1988). In particular, this is true if the distribution of the innovations has a Lebesgue density $f$ and we take $\psi=-f^{\prime} / f$, i.e. if we use ML-estimates. The gain in efficiency carries over to the estimators $\hat{\mathbf{r}}$ and $\hat{a}(p)$, which are smooth functions of $\hat{a}^{M}=\hat{a}^{M}\left(p_{M}\right)$. 
It is not possible to use the M-estimators $\hat{a}^{M}(p)$ directly as estimators for $a(p)$, because, in general, they are not even consistent. Therefore, we have to use the detour of calculating $\hat{\mathbf{r}}$ from $\hat{a}^{M}\left(p_{M}\right)$ and then define $\hat{a}(p)$ as in (3.2) to get robust and consistent estimates of $a(p)$ for $p \leq p_{M}$. For a different approach where the quadratic loss function in (2.2) is replaced by some loss function $L_{\psi}$ and where the optimal parameters may be estimated directly by the M-estimators, see Behrens (1990).

To avoid too much technical details, we consider in the following two sections only the easiest case where $\hat{r}_{h}=\tilde{r}_{h}, h \leq p(n)$, are the empirical autocorrelations and $\hat{a}(p)=\tilde{a}(p)$ are the Yule-Walker estimates. A theoretical investigation of the asymptotic properties of our bootstrap order selection procedure when $\hat{\mathbf{r}}$ corresponds to some M-estimator $\hat{a}^{M}\left(p_{M}\right)$ is considerably more involved and will be the subject of a forthcoming paper, see also Moser (1997).

\section{Bootstrap Order Selection}

Let us first briefly introduce the bootstrap principle for $\mathrm{AR}(\infty)$-processes which will be applied in the following. For a fuller account the interested reader is referred to Kreiss (1988, 1997) and Bühlmann (1997).

Given the observations $X_{1}, \ldots, X_{n}$ we fit an autoregression of "large" order $p_{0}=p_{0}(n) \geq$ $p(n)$ and compute approximate innovations

$$
\varepsilon_{t}\left(\tilde{a}\left(p_{0}\right)\right)=X_{t}-\tilde{a}\left(p_{0}\right)^{T} X_{t-1}\left(p_{0}\right), \quad t=p_{0}+1, \ldots, n,
$$

with empirical and centered (around mean 0) empirical (cumulative) distribution functions $\tilde{F}_{n}, \tilde{F}_{n}^{c}$, respectively.

Now suppose that the process $\left(\varepsilon_{t}^{*}: t \in \mathbb{Z}\right)$ consists of i.i.d. random variables with distribution function $\tilde{F}_{n}^{c}$. This ensures $E^{*} \varepsilon_{t}^{*}=0$, where $E^{*}$ denotes the conditional expectation $E\left[\cdot \mid X_{1}, \ldots, X_{n}\right]$. It is well-known known that $1-\sum_{\ell=1}^{p_{0}} \tilde{a}_{\ell}\left(p_{0}\right) z^{\ell}$ has no zeros in the closed unit disk and therefore $\mathbf{j}\left(\tilde{a}\left(p_{0}\right)\right)$ has an inverse $\tilde{\boldsymbol{\alpha}}\left(p_{0}\right)$ in $\ell^{1}$ with respect to convolution; cf. Brockwell and Davis (1991), p. 240. Hence we may define the bootstrap process $\left(X_{t}^{*}: t \in \mathbb{Z}\right)$ as an autoregression of order $p_{0}$ with coefficients $\mathbf{a}^{*}=\tilde{a}\left(p_{0}\right)$ and white noise process $\left(\varepsilon_{t}^{*}: t \in \mathbb{Z}\right)$, i.e.

$$
X_{t}^{*}=\sum_{\ell=1}^{p_{0}} \tilde{a}_{\ell}\left(p_{0}\right) X_{t-\ell}^{*}+\varepsilon_{t}^{*}=\sum_{\ell=0}^{\infty} \tilde{\alpha}_{\ell}\left(p_{0}\right) \varepsilon_{t-\ell}^{*}, \quad t \in \mathbb{Z} .
$$

For later reference we note some asymptotic properties of the bootstrap construction. Assuming $p_{0}(n) \rightarrow \infty$ and $p_{0}(n)^{4} / n \rightarrow 0$ we have from Kreiss (1997) $E^{*} \varepsilon^{* k} \rightarrow E \varepsilon^{k}$ in probability, $k=2,4$, (Proposition 3.1) and $\left\|\boldsymbol{\alpha}-\tilde{\boldsymbol{\alpha}}\left(p_{0}\right)\right\|_{1} \rightarrow 0$ in probability (Lemma 8.2 and 8.3). As the autocovariance function $\gamma$ is a continuous function of $E \varepsilon_{0}^{2} \in \mathbb{R}$ and $\alpha \in \ell^{1}$ this implies $\left\|\gamma-\gamma^{*}\right\|_{1} \rightarrow 0$ in probability for the autocovariance function $\gamma^{*}$ with components $\gamma_{h}^{*}=E^{*} X_{t}^{*} X_{t+h}^{*}$ of the bootstrap autoregressive process. 
Of course $\gamma^{*}$ is closely related to the empirical autocovariance function $\tilde{\gamma}$. In fact, the corresponding autocorrelation functions $\mathbf{r}^{*}=\frac{1}{\gamma_{0}^{*}} \gamma^{*}$ and $\tilde{\mathbf{r}}=\frac{1}{\tilde{\gamma}_{0}} \tilde{\gamma}$ coincide up to lag $p_{0}$, where both $\gamma_{0}^{*}$ and $\tilde{\gamma}_{0}$ converge to $\gamma_{0}$ in probability. In particular, we have

$$
a^{*}(p)=\Gamma^{*}(p)^{-1} \gamma^{*}(p)=\tilde{\Gamma}(p)^{-1} \tilde{\gamma}(p)=\tilde{a}(p), \quad 1 \leq p \leq p_{0} .
$$

Let $\tilde{\gamma}^{*}$ and $\tilde{a}^{*}(p)$ be exactly defined as $\tilde{\gamma}$ and $\tilde{a}(p)$, with $\left(X_{1}, \ldots, X_{n}\right)$ replaced by $\left(X_{1}^{*}, \ldots, X_{n}^{*}\right)$, the bootstrap observations.

We propose to replace $E\|\tilde{a}(p)-a(p)\|_{\Gamma(p)}^{2}$ in the definition of the order selection $P_{1}^{\prime}(n)$ (cf. (2.9)) by its bootstrap approximation

$$
E^{*}\left\|\tilde{a}^{*}(p)-a^{*}(p)\right\|_{\Gamma^{*}(p)}^{2}=E^{*}\left\|\tilde{a}^{*}(p)-\tilde{a}(p)\right\|_{\Gamma^{*}(p)}^{2} \cdot
$$

To avoid technical problems, we will work with the order selection $P_{1}(n)$ instead of $P_{1}^{\prime}(n)$, so we will use the bootstrap analogon

$$
S_{n}^{*}(p)=E^{*}\left\|\tilde{\gamma}^{*}(p)-\tilde{\Gamma}^{*}(p) \tilde{a}(p)\right\|_{\Gamma^{*}(p)^{-1}}^{2} \cdot
$$

of $S_{n}(p)$, as we already mentioned in Section 2, cf. (2.7).

Hence we define the bootstrap order selection as

$$
P_{B}(n):=\underset{1 \leq p \leq p(n)}{\arg \min }\left\{\tilde{\gamma}_{0}-\tilde{a}(p)^{T} \tilde{\gamma}(p)+2 \cdot S_{n}^{*}(p)\right\}
$$

We remark that the whole procedure resulting in the order selection $P_{B}(n)$ can be done with general autocovariance estimates $\hat{\gamma}$ and the corresponding sample prediction coefficients $\hat{a}(p)$, given by (3.2). We restrict ourselves to the sample autocovariances $\tilde{\gamma}$ and the Yule-Walker estimates $\tilde{a}(p)$ only to simplify the proofs. One of our main results is as follows.

Theorem 4.1 : Let $\{p(n): n \in \mathbb{I}\}$ and $\left\{p_{0}(n): n \in \mathbb{N}\right\}$ be two sequences of integers with $p(n) \leq p_{0}(n)$ for all $n \in \mathbb{N}$ and $p(n) \rightarrow \infty, p_{0}(n)^{4} / n \rightarrow 0$ as $n \rightarrow \infty$. Then we have for $S_{n}(p), S_{n}^{*}(p)$ defined in (D.8) and (4.1)

$$
\max _{1 \leq p \leq p(n)}\left\{\frac{n}{p}\left|S_{n}^{*}(p)-S_{n}(p)\right|\right\}=o_{P}(1) .
$$

All proofs are collected in Section 7.

As will be seen in the proof of Theorem $5.1, \frac{n}{p} S_{n}(p) \rightarrow \sigma^{2}$ if $p$ is "large". Hence Theorem 4.1 basically maintains that the difference between $S_{n}(p)$ and its bootstrap approximation $S_{n}^{*}(p)$ tends faster to 0 than $S_{n}(p)$ itself, i.e. $S_{n}(p)^{-1}\left|S_{n}^{*}(p)-S_{n}(p)\right|=o_{P}(1)$, uniformly in all "large" $p$. If $p$ is "small", then $\tilde{\gamma}_{0}-\tilde{a}(p)^{T} \tilde{\gamma}(p)$ will be the dominating term in $\tilde{\gamma}_{0}-\tilde{a}(p)^{T} \tilde{\gamma}(p)-2 S_{n}(p)$, the expression minimized by $P_{1}(n)$, cf. (2.7). In this case the relative difference $S_{n}(p)^{-1}\left|S_{n}^{*}(p)-S_{n}(p)\right|$ will be of secondary importance as long as the absolute difference tends to 0 fast enough. 


\section{Asymptotic Properties of the Bootstrap Order Selection}

In this section we deal with some asymptotic properties of the proposed bootstrap order selection $P_{B}(n)$ defined by (4.2):

$$
P_{B}(n)=\underset{1 \leq p \leq p(n)}{\arg \min }\left\{\tilde{\gamma}_{0}-\tilde{a}(p)^{T} \tilde{\gamma}(p)+2 \cdot S_{n}^{*}(p)\right\}
$$

The first part of the criterion function can be written in the more familiar way

$$
\tilde{\gamma}_{0}-\tilde{a}(p)^{T} \tilde{\gamma}(p)=\frac{1}{n} \sum_{t=p_{0}(n)+1}^{n}\left(X_{t}-\tilde{a}(p)^{T} X_{t-1}(p)\right)^{2}=: \tilde{\sigma}_{n}^{2}(p),
$$

if we assume that, for the sake of simplicity, we slightly modify the definitions of Section 3 to

$$
\tilde{\gamma}_{h}:=\frac{1}{n} \sum_{t=p(n)+1}^{n} X_{t} X_{t-h}, \quad h=0,1,2, \ldots, p(n)
$$

and

$$
\tilde{a}(p):=\left[\frac{1}{n} \sum_{t=p(n)+1}^{n} X_{t-1}(p) X_{t-1}(p)^{T}\right]^{-1}\left(\tilde{\gamma}_{1}, \ldots, \tilde{\gamma}_{p}\right)^{T} \equiv \tilde{\Gamma}(p)^{-1} \tilde{\gamma}(p),
$$

which are essentially the usual Yule-Walker estimators up to asymptotically negligible terms.

Now we state the main result of this section. Again, the proof is deferred to Section 7.

Theorem 5.1 : Under the assumptions of Theorem 4.1 we have

$$
\max _{1 \leq p \leq p(n)} \frac{\left|S_{n}^{*}(p)-\frac{p}{n} \sigma^{2}\right|}{\frac{p}{n}+\frac{\sigma^{2}(p)-\sigma^{2}}{\sigma^{2}}}=o_{P}(1)
$$

where $\sigma^{2}(p)=E\left(X_{t}-a(p)^{T} X_{t-1}(p)\right)^{2}$.

¿From this result we can derive an interesting property of the bootstrap order selection, observing that

$$
\max _{p} \frac{\frac{p}{n}\left|\sigma^{2}-\sigma^{2}(p)\right|}{\frac{p}{n}+\frac{\sigma^{2}(p)-\sigma^{2}}{\sigma^{2}}} \leq \max _{p} \frac{\frac{p}{n}\left|\sigma^{2}-\sigma^{2}(p)\right|}{\frac{\sigma^{2}(p)-\sigma^{2}}{\sigma^{2}}}=\frac{p(n)}{n} \sigma^{2}=o(1)
$$

and

$$
\begin{array}{r}
\max _{p} \frac{\frac{p}{n}\left|\tilde{\sigma}_{n}^{2}(p)-\sigma^{2}(p)\right|}{\frac{p}{n}+\frac{\sigma^{2}(p)-\sigma^{2}}{\sigma^{2}}} \leq \max _{p}\left|\tilde{\sigma}_{n}^{2}(p)-\sigma^{2}(p)\right| \\
\leq \max _{p}\left|\tilde{\sigma}_{n}^{2}(p)-\frac{1}{n} \sum_{t=p(n)+1}^{n}\left(X_{t}-a(p)^{T} X_{t-1}(p)\right)^{2}\right|
\end{array}
$$




$$
\begin{aligned}
& +\max _{p}\left|\frac{1}{n} \sum_{t=p(n)+1}^{n}\left(X_{t}-a(p)^{T} X_{t-1}(p)\right)^{2}-\sigma^{2}(p)\right| \\
= & \max _{p}\|\tilde{a}(p)-a(p)\|_{\tilde{\Gamma}(p)}^{2}+\max _{p}\left|\frac{1}{n} \sum_{t}\left(X_{t}-a(p)^{T} X_{t-1}(p)\right)^{2}-\sigma^{2}(p)\right| \\
= & o_{P}(1)
\end{aligned}
$$

as the first expression is $o_{P}(1)$ by (7.6) and (7.9) and the second expression by an application of Lemma 7.1 to the process $Y_{t}=X_{t}-a(p)^{T} X_{t-1}(p)$.

This together with Theorem 5.1 and with Theorem 7.4.7 of Deistler and Hannan (1988) implies that we have uniformly in $p \in\{1, \ldots, p(n)\}$

$$
\begin{aligned}
& \log \left\{\tilde{\sigma}_{n}^{2}(p)+2 \cdot S_{n}^{*}(p)\right\} \\
= & \log \tilde{\sigma}_{n}^{2}(p)+\log \left(1+2 \cdot \frac{S_{n}^{*}(p)}{\tilde{\sigma}_{n}^{2}(p)}\right) \\
= & \log \tilde{\sigma}_{n}^{2}(p)+\log \left(1+\frac{p}{n}\left[\frac{2}{\tilde{\sigma}_{n}^{2}(p)}\left\{\frac{n}{p} S_{n}^{*}(p)-\tilde{\sigma}_{n}^{2}(p)\right\}+2\right]\right) \\
= & \log \tilde{\sigma}_{n}^{2}(p)+2 \frac{p}{n}+\frac{p}{n}\left[\frac{2}{\tilde{\sigma}_{n}^{2}(p)}\left\{\frac{n}{p} S_{n}^{*}(p)-\tilde{\sigma}_{n}^{2}(p)\right\}+o_{P}(1)\right] \\
= & \log \dot{\sigma}_{n}^{2}+\left\{\frac{p}{n}+\frac{\sigma^{2}(p)-\sigma^{2}}{\sigma^{2}}\right\}\left(1+o_{P}(1)\right) \\
& +\frac{p}{n}\left[\frac{2}{\tilde{\sigma}_{n}^{2}(p)}\left\{\frac{n}{p} S_{n}^{*}(p)-\tilde{\sigma}_{n}^{2}(p)\right\}+o_{P}(1)\right] \\
= & \log \dot{\sigma}_{n}^{2}+\left\{\frac{p}{n}+\frac{\sigma^{2}(p)-\sigma^{2}}{\sigma^{2}}\right\}\left(1+o_{P}(1)\right) \\
& +\frac{2}{\tilde{\sigma}_{n}^{2}(p)} \cdot \frac{S_{n}^{*}(p)-p / n \tilde{\sigma}_{n}^{2}(p)}{p / n+\frac{\sigma^{2}(p)-\sigma^{2}}{\sigma^{2}}}\left\{\frac{p}{n}+\frac{\sigma^{2}(p)-\sigma^{2}}{\sigma^{2}}\right\} \\
= & \log \dot{\sigma}_{n}^{2}+\left\{\frac{p}{n}+\frac{\sigma^{2}(p)-\sigma^{2}}{\sigma^{2}}\right\}\left(1+o_{P}(1)\right),
\end{aligned}
$$

where $\dot{\sigma}_{n}^{2}$ is defined in Deistler and Hannan (1988), above Theorem 7.4.5, and is equal to

$$
\dot{\sigma}_{n}^{2}=\frac{1}{n} \sum_{t=1}^{n} \varepsilon_{t}^{2}, n \in I N
$$

Summarizing we obtain from Theorem 5.1 the following expansion, which holds uniformly in $p \in\{1, \ldots, p(n)\}$

$$
\log \left\{\tilde{\sigma}_{n}^{2}(p)+2 \cdot S_{n}^{*}(p\}=\log \dot{\sigma}_{n}^{2}+\left\{\frac{p}{n}+\frac{\sigma^{2}(p)-\sigma^{2}}{\sigma^{2}}\right\}\left(1+o_{P}(1)\right) .\right.
$$

This is exactly the same expansion as Deistler and Hannan obtained for the AIC, cf. Deistler and Hannan (1988), Theorem 7.4.7. In other words, the considerations given 
below Theorem 7.4.7 in Deistler and Hannan (1988) hold also true for the bootstrap order selection.

Remark . (i) Shibata has a result similar to Theorem 5.1 in his paper, cf. Shibata (1980) Lemma 7.1, but we can dispend with the assumption of normality.

(ii) From Theorem 5.1 we obtain exactly along the lines of Section 4 in Shibata (1980) the asymptotic efficiency of the bootstrap order selection under the same assumptions as in Shibata. The concept of asymptotic efficiency is also defined by Shibata.

Following the arguments given in Deistler and Hannan (1988), p. 333/334, we obtain exactly along the same lines and under the same assumption that

$$
\frac{P_{B}(n)}{\operatorname{argmin}_{1 \leq p \leq p(n)}\left(\frac{p}{n}+\frac{\sigma^{2}(p)-\sigma^{2}}{\sigma^{2}}\right)} \rightarrow 1 \quad \text { in probability. }
$$

In the next Section we report some simulation results for the bootstrap order selection in comparison with other order selection procedures.

\section{Simulations}

Let us consider the following two order selection procedures for a simulation study. The argument of the minimum (argmin) is in both cases computed over the range $\{1, \ldots, p(n)\}$.

$$
\begin{aligned}
\mathbf{A I C} & =\operatorname{argmin}_{p}\left\{\hat{\sigma}_{n}^{2}(p) \cdot\left(1+\frac{2 p}{n}\right)\right\} \\
\mathbf{P}_{\mathbf{B}} & =\operatorname{argmin}_{p}\left\{\hat{\sigma}_{n}^{2}(p)+2 \cdot E^{*}\left\|\hat{a}^{*}(p)-\tilde{a}(p)\right\|_{\tilde{R}(p)}^{2}\right\} .
\end{aligned}
$$

In all cases

$$
\hat{\sigma}_{n}^{2}(p)=1-\sum_{\ell=1}^{p} \hat{a}_{\ell} \hat{r}_{\ell}
$$

and $\hat{r}_{h}$ denotes an estimator of the autocorrelation at lag $h$, which does not necessarily have to coincide with the empirical autocorrelation $\tilde{r}_{h}$ of the observations. This deviates slightly from the preceding sections, where we preferred to work with the autocovariances in order to simplify the proofs, and obviates the need for an M-estimator of $\gamma_{0}$. The AIC goes back to Akaike (1973a,b, 1974). $P_{B}$ denotes the bootstrap order selection proposed in Section 4 of the present paper. Note that for the theoretical investigation we used a slightly modified version of $P_{B}$. 


\begin{tabular}{|c||c|c|c|c|c|c|c|c|}
\hline criterion & $\mathrm{p}=1$ & $\mathrm{p}=2$ & $\mathrm{p}=3$ & $\mathrm{p}=4$ & $\mathrm{p}=5$ & $\mathrm{p}=6$ & $\mathrm{p}=7$ & $\mathrm{p}=8$ \\
\hline \hline AIC & 2 & 0 & 67 & 14 & 8 & 3 & 3 & 3 \\
\hline $\mathbf{A I C}$ & 3 & 0 & 72 & 16 & 4 & 4 & 1 & 0 \\
\hline $\mathbf{A I C}$ & 4 & 0 & 69 & 13 & 5 & 6 & 2 & 1 \\
\hline $\mathbf{P}_{\mathbf{B}}, \psi_{\text {id }}$ & 1 & 1 & 68 & 13 & 6 & 4 & 2 & 5 \\
\hline $\mathbf{P}_{\mathbf{B}}, \psi_{\text {id }}$ & 5 & 1 & 70 & 12 & 5 & 3 & 2 & 2 \\
\hline $\mathbf{P}_{\mathbf{B}}, \psi_{\text {id }}$ & 5 & 0 & 63 & 14 & 9 & 3 & 3 & 3 \\
\hline $\mathbf{P}_{\mathbf{B}}, \psi_{\text {Huber }}$ & 1 & 1 & 68 & 10 & 7 & 8 & 3 & 2 \\
\hline $\mathbf{P}_{\mathbf{B}}, \psi_{\text {Huber }}$ & 3 & 0 & 66 & 17 & 3 & 6 & 2 & 3 \\
\hline $\mathbf{P}_{\mathbf{B}}, \psi_{\text {Huber }}$ & 3 & 0 & 65 & 12 & 9 & 4 & 3 & 4 \\
\hline
\end{tabular}

Table 6.1

frequencies of selected orders (100 repetitions)

model (6.3), normal innovations, sample size $=100, p(n)=8$

The simulations we are going to report are based on the following three stationary time series models

$$
\begin{aligned}
& X_{t}=0.64 \cdot X_{t-1}-0.19 \cdot X_{t-2}+0.39 \cdot X_{t-3}+\varepsilon_{t} \\
& X_{t}=-X_{t-2}-0.1 \cdot X_{t-4}+\varepsilon_{t} \\
& X_{t}=-0.5 \cdot X_{t-2}+0.5 \cdot \varepsilon_{t-1}+\varepsilon_{t} .
\end{aligned}
$$

The first two models are of finite autoregressive order, while the ARMA(2,1)-model (6.5) possesses an autoregressive representation of infinite order.

For the innovations $\varepsilon_{t}$ we use the following distributions

$$
\begin{array}{lll}
\varepsilon_{1} & \sim \mathcal{N}(0,1) \quad \text { normally distributed innovations } \\
\varepsilon_{1} \sim 0.8 \mathcal{N}(0,1)+0.2 \mathcal{N}(0,25) & \text { contaminated innovations } \\
\varepsilon_{1} \sim 0.5(\mathcal{N}(-3,1)+\mathcal{N}(3,1)) & \text { bimodal normal innovations }
\end{array}
$$

The AIC is always computed using least squares parameter estimates, for which this criterion is designed. However, changing the parameter estimates does not affect the AIC essentially. The bootstrap order selection $P_{B}$ is computed for different M-estimators. Here we make use of $\psi_{\text {id }}(x)=x$, corresponding to least squares, and $\psi_{\text {Huber }}(x)=-\mathbf{1}_{(x<-1)}+$ $x \cdot \mathbf{1}_{(-1 \leq x \leq 1)}+\mathbf{1}_{(x>1)}$.

We report on the simulated behaviour of the procedures on two different random samples of 100 time series each in order to give an impression of the stochastic fluctuation of the results. 


\begin{tabular}{|c||c|c|c|c|c|c|c|c|}
\hline criterion & $\mathrm{p}=1$ & $\mathrm{p}=2$ & $\mathrm{p}=3$ & $\mathrm{p}=4$ & $\mathrm{p}=5$ & $\mathrm{p}=6$ & $\mathrm{p}=7$ & $\mathrm{p}=8$ \\
\hline \hline $\mathbf{A I C}$ & 0 & 71 & 7 & 12 & 4 & 2 & 0 & 4 \\
\hline $\mathbf{A I C}$ & 0 & 61 & 11 & 15 & 7 & 2 & 2 & 2 \\
\hline $\mathbf{A I C}$ & 0 & 65 & 13 & 13 & 3 & 4 & 1 & 1 \\
\hline $\mathbf{P}_{\mathbf{B}}, \psi_{\text {id }}$ & 0 & 68 & 5 & 16 & 5 & 2 & 2 & 2 \\
\hline $\mathbf{P}_{\mathbf{B}}, \psi_{\text {id }}$ & 0 & 59 & 10 & 13 & 6 & 3 & 3 & 6 \\
\hline $\mathbf{P}_{\mathbf{B}}, \psi_{\text {id }}$ & 0 & 70 & 6 & 17 & 2 & 4 & 0 & 1 \\
\hline $\mathbf{P}_{\mathbf{B}}, \psi_{\text {opt }}$ & 0 & 55 & 3 & 37 & 4 & 0 & 1 & 0 \\
\hline $\mathbf{P}_{\mathbf{B}}, \psi_{\text {opt }}$ & 0 & 67 & 0 & 30 & 1 & 1 & 0 & 1 \\
\hline $\mathbf{P}_{\mathbf{B}}, \psi_{\text {opt }}$ & 0 & 62 & 6 & 27 & 5 & 0 & 0 & 0 \\
\hline
\end{tabular}

Table 6.2

frequencies of selected orders (100 repetitions)

model (6.4), bimodal innovations, sample size $=100, \mathrm{p}(\mathrm{n})=8$

Tables 6.1 gives the results for model (6.3). From this table it can be seen that the results for normally distributed observations do not differ very much. This means that the proposed bootstrap order selection procedure behaves more or less like the AIC for standard situations. For non-normally distributed innovations the situation is quite different. To demonstrate this let us first consider model (6.4) with bimodal normally distributed innovations and sample sizes $n=100$ (cf. Table 6.2) and $n=200$ (cf. Table 6.3). Here we make use of the asymptotically optimal choice of the $\psi$-function, namely $\psi$ equal to the logarithmic derivative of the underlying density, i.e. $\psi=-f^{\prime} / f$. Additionally we present results for the least-squares estimator, i.e. $\psi(x)=x$.

\begin{tabular}{|c||c|c|c|c|c|c|c|c|}
\hline criterion & $\mathrm{p}=1$ & $\mathrm{p}=2$ & $\mathrm{p}=3$ & $\mathrm{p}=4$ & $\mathrm{p}=5$ & $\mathrm{p}=6$ & $\mathrm{p}=7$ & $\mathrm{p}=8$ \\
\hline \hline AIC & 0 & 55 & 8 & 26 & 4 & 2 & 0 & 5 \\
\hline AIC & 0 & 57 & 2 & 32 & 3 & 2 & 0 & 4 \\
\hline AIC & 0 & 45 & 5 & 31 & 6 & 7 & 4 & 2 \\
\hline $\mathbf{P}_{\mathbf{B}}, \psi_{\text {id }}$ & 0 & 51 & 8 & 26 & 4 & 6 & 4 & 1 \\
\hline $\mathbf{P}_{\mathbf{B}}, \psi_{\text {id }}$ & 0 & 52 & 7 & 24 & 10 & 4 & 2 & 1 \\
\hline $\mathbf{P}_{\mathbf{B}}, \psi_{\text {id }}$ & 0 & 59 & 3 & 28 & 7 & 0 & 1 & 2 \\
\hline $\mathbf{P}_{\mathbf{B}}, \psi_{\text {opt }}$ & 0 & 9 & 0 & 79 & 7 & 2 & 3 & 0 \\
\hline $\mathbf{P}_{\mathbf{B}}, \psi_{\text {opt }}$ & 0 & 14 & 0 & 74 & 9 & 2 & 0 & 1 \\
\hline $\mathbf{P}_{\mathbf{B}}, \psi_{\text {opt }}$ & 0 & 5 & 0 & 81 & 9 & 3 & 0 & 2 \\
\hline
\end{tabular}

Table 6.3

frequencies of selected orders (100 repetitions)

model (6.4), bimodal innovations, sample size $=200, p(n)=8$ 


\begin{tabular}{|c||c|c|c|c|c|c|c|c|c|c|}
\hline criterion & $\mathrm{p}=1$ & $\mathrm{p}=2$ & $\mathrm{p}=3$ & $\mathrm{p}=4$ & $\mathrm{p}=5$ & $\mathrm{p}=6$ & $\mathrm{p}=7$ & $\mathrm{p}=8$ & $\mathrm{p}=9$ & $\mathrm{p}=10$ \\
\hline \hline AIC & 0 & 1 & 22 & 44 & 18 & 11 & 0 & 0 & 1 & 3 \\
\hline AIC & 0 & 1 & 17 & 42 & 20 & 10 & 5 & 3 & 1 & 1 \\
\hline AIC & 0 & 0 & 18 & 52 & 17 & 3 & 4 & 5 & 0 & 1 \\
\hline $\mathbf{P}_{\mathbf{B}}, \psi_{\text {Huber }}$ & 0 & 0 & 4 & 31 & 29 & 21 & 8 & 2 & 4 & 1 \\
\hline $\mathbf{P}_{\mathbf{B}}, \psi_{\text {Huber }}$ & 0 & 0 & 3 & 30 & 29 & 18 & 7 & 5 & 5 & 3 \\
\hline $\mathbf{P}_{\mathbf{B}}, \psi_{\text {Huber }}$ & 0 & 0 & 4 & 41 & 30 & 11 & 8 & 2 & 4 & 0 \\
\hline $\mathbf{P}_{\mathbf{B}}, \psi_{\text {opt }}$ & 0 & 0 & 2 & 40 & 38 & 6 & 8 & 3 & 2 & 1 \\
\hline $\mathbf{P}_{\mathbf{B}}, \psi_{\text {opt }}$ & 0 & 0 & 1 & 39 & 40 & 13 & 2 & 2 & 0 & 3 \\
\hline $\mathbf{P}_{\mathbf{B}}, \psi_{\text {opt }}$ & 0 & 0 & 3 & 37 & 29 & 11 & 9 & 3 & 8 & 0 \\
\hline
\end{tabular}

Table 6.4

frequencies of selected orders (100 repetitions)

model (6.5), contaminated innovations, sample size $=200, p(n)=200$

It can be seen clearly, especially from Table 6.3, that the bootstrap order selection using the asymptotically optimal $\psi$-function tends to select the true order with much higher probability. This is due to the fact that M-estimators with this $\psi$-function have much smaller variance than, for example, the least squares estimator used in the construction of the AIC.

Finally, for the ARMA(2,1)-model (6.5) we again demonstrate the behaviour of the bootstrap order selection for two different M-estimators $\left(\psi_{\text {Huber }}\right.$ and $\left.\psi_{\text {opt }}=-f^{\prime} / f\right)$ and contaminated innovations (cf. Table 6.4). The precision of the parameter estimates increases from the Huber M-estimator to M-estimates with asymptotically optimal score-function, which implies the desired property that the $P_{B}$ tends to higher orders for the autoregressive fit.

Acknowledgement. Parts of the research presented in this paper was done while the second author enjoyed the hospitality of the Sonderforschungsbereich 123 at the University of Heidelberg which is gratefully acknowledged. The third author acknowledges the support of the DFG-project MA 1026/6-1.

\section{Proofs}

The proof of Theorem 4.1 will be based on the following approximation lemma, which is of interest on its own.

Lemma 7.1 : Under the assumptions of Theorem 4.1 we have

$$
\begin{aligned}
\max _{1 \leq h, k \leq n}\left|\operatorname{Cov}^{*}\left(\tilde{\gamma}_{h}^{*}, \tilde{\gamma}_{k}^{*}\right)-\operatorname{Cov}\left(\tilde{\gamma}_{h}, \tilde{\gamma}_{k}\right)\right| & =o_{P}\left(n^{-1}\right), \\
\max _{1 \leq h, k \leq n}\left|\operatorname{Cov}\left(\tilde{\gamma}_{h}, \tilde{\gamma}_{k}\right)\right| & =O\left(n^{-1}\right) .
\end{aligned}
$$


Proof : We will show the following inequality

$$
\begin{gathered}
\max _{1 \leq h, k \leq n}\left|\operatorname{Cov}^{*}\left(\tilde{\gamma}_{h}^{*}, \tilde{\gamma}_{k}^{*}\right)-\operatorname{Cov}\left(\tilde{\gamma}_{h}, \tilde{\gamma}_{k}\right)\right| \leq \frac{3}{n}\left\|\boldsymbol{\alpha}-\boldsymbol{\alpha}^{*}\right\|_{1}\left(\|\boldsymbol{\alpha}\|_{1}+\left\|\boldsymbol{\alpha}^{*}\right\|_{1}\right)^{3} E \varepsilon_{0}^{4} \\
+\frac{3}{n}\left\|\boldsymbol{\alpha}^{*}\right\|_{1}^{4}\left(\left|\left(E \varepsilon_{0}^{2}\right)^{2}-\left(E^{*} \varepsilon_{0}^{* 2}\right)^{2}\right|+\left|E \varepsilon_{0}^{4}-E^{*} \varepsilon_{0}^{* 4}\right|\right)
\end{gathered}
$$

where $\boldsymbol{\alpha}^{*}=\tilde{\boldsymbol{\alpha}}\left(p_{0}\right)=\mathbf{j}\left(\tilde{a}\left(p_{0}\right)\right)^{-1}$. The asymptotic properties of the bootstrap construction mentioned in Section 4 will then imply (7.1), and (7.2) will follow from (7.3) by setting $\alpha^{*}=\gamma^{*}=$ O.

The proof of (7.3) will be based on the $\mathrm{MA}(\infty)$-representation of the process $\left(X_{t}\right)$, which yields the following formula for the empirical autocovariances:

$$
\begin{aligned}
\tilde{\gamma}_{h}-E \tilde{\gamma}_{h} & =\frac{1}{n} \sum_{t=1}^{n-h} \sum_{j, \ell=0}^{\infty} \alpha_{j} \alpha_{\ell} \varepsilon_{t-j} \varepsilon_{t+h-\ell}+\frac{1}{n} \sum_{t=1}^{n-h} \sum_{j=0}^{\infty} \alpha_{j} \alpha_{j+h}\left(\varepsilon_{t-j}^{2}-E \varepsilon_{0}^{2}\right) \\
=: & V(h)+W(h),
\end{aligned}
$$

where the dagger indicates that summation takes place only over those pairs $(j, \ell)$ with $\ell \neq j+h$. For any $h_{1}, h_{2}$, the sums $V\left(h_{1}\right)$ and $W\left(h_{2}\right)$ are uncorrelated which implies $\operatorname{Cov}\left(\tilde{\gamma}_{h_{1}}, \tilde{\gamma}_{h_{2}}\right)=E V\left(h_{1}\right) V\left(h_{2}\right)+E W\left(h_{1}\right) W\left(h_{2}\right)$. Furthermore

$$
\begin{aligned}
E V\left(h_{1}\right) V\left(h_{2}\right) & =\frac{1}{n^{2}} E \prod_{i=1}^{2}\left(\sum_{t=1}^{n-h_{i}} \sum_{j, \ell=0}^{\infty}+\alpha_{j} \alpha_{\ell} \varepsilon_{t-j} \varepsilon_{t+h_{i}-\ell}\right) \\
& =\frac{1}{n^{2}} \sum_{j_{1}, \ell_{1}=0}^{\infty} \sum_{j_{2}, \ell_{2}=0}^{\infty} \dagger \prod_{i=1}^{2}\left(\alpha_{j_{i}} \alpha_{\ell_{i}}\right) \sum_{t_{1}=1}^{n-h_{1}} \sum_{t_{2}=1}^{n-h_{2}} E \prod_{i=1}^{2}\left(\varepsilon_{t_{i}-j_{i}} \varepsilon_{t_{i}+h_{i}-\ell_{i}}\right) .
\end{aligned}
$$

As $j_{i} \neq h_{i}+\ell_{i}$, the last expectation equals zero unless $t_{1}-j_{1}=t_{2}-j_{2}$ or $t_{1}-j_{1}=t_{2}+h_{2}-\ell_{2}$, in which case it may be $\left(E \varepsilon_{0}^{2}\right)^{2}$ instead of zero. Hence the double sum over $t_{1}, t_{2}$ reduces to two single sums over $t_{1}$. Taking the difference $E V\left(h_{1}\right) V\left(h_{2}\right)-E^{*} V^{*}\left(h_{1}\right) V^{*}\left(h_{2}\right)$ we first replace $\boldsymbol{\alpha}$ by $\boldsymbol{\alpha}^{*}$ to get

$$
\begin{aligned}
& \left|\frac{1}{n^{2}} \sum_{j_{1}, \ell_{1}=0}^{\infty}+\sum_{j_{2}, \ell_{2}=0}^{\infty}+\left(\prod_{i=1}^{2} \alpha_{j_{i}} \alpha_{\ell_{i}}-\prod_{i=1}^{2} \alpha_{j_{i}}^{*} \alpha_{\ell_{i}}^{*}\right) \sum_{t_{1}=1}^{n-h_{1}} \sum_{t_{2}=1}^{n-h_{2}} E \prod_{i=1}^{2}\left(\varepsilon_{t_{i}-j_{i}} \varepsilon_{t_{i}+h_{i}-\ell_{i}}\right)\right| \\
\leq & \frac{2}{n}\left\|\boldsymbol{\alpha}-\boldsymbol{\alpha}^{*}\right\|_{1}\left(\|\boldsymbol{\alpha}\|_{1}+\left\|\boldsymbol{\alpha}^{*}\right\|_{1}\right)^{3}\left(E \varepsilon_{0}^{2}\right)^{2} .
\end{aligned}
$$

In a second step the innovations $\varepsilon_{t}$ are exchanged for the bootstrap innovations $\varepsilon_{t}^{*}$, yielding

$$
\begin{aligned}
& \left|\frac{1}{n^{2}} \sum_{j_{1}, \ell_{1}=0}^{\infty} \dagger \sum_{j_{2}, \ell_{2}=0}^{\infty}+\prod_{i=1}^{2} \alpha_{j_{i}}^{*} \alpha_{\ell_{i}}^{*} \sum_{t_{1}=1}^{n-h_{1}} \sum_{t_{2}=1}^{n-h_{2}} E \prod_{i=1}^{2} \varepsilon_{t_{i}-j_{i}} \varepsilon_{t_{i}+h_{i}-\ell_{i}}-E^{*} \prod_{i=1}^{2} \varepsilon_{t_{i}-j_{i}}^{*} \varepsilon_{t_{i}+h_{i}-\ell_{i}}^{*}\right| \\
& \quad \leq \frac{2}{n}\left\|\boldsymbol{\alpha}^{*}\right\|_{1}^{4}\left|\left(E \varepsilon_{0}^{2}\right)^{2}-\left(E \varepsilon_{0}^{* 2}\right)^{2}\right| .
\end{aligned}
$$


A bound for $E V\left(h_{1}\right) V\left(h_{2}\right)-E^{*} V^{*}\left(h_{1}\right) V^{*}\left(h_{2}\right)$ is obtained by adding the bounds in (7.4) and (7.5). A similar calculation leads to

$$
\begin{gathered}
\left|E W\left(h_{1}\right) W\left(h_{2}\right)-E^{*} W^{*}\left(h_{1}\right) W^{*}\left(h_{2}\right)\right| \leq \frac{1}{n}\left\|\boldsymbol{\alpha}-\boldsymbol{\alpha}^{*}\right\|_{1}\left(\|\boldsymbol{\alpha}\|_{1}+\left\|\boldsymbol{\alpha}^{*}\right\|_{1}\right)^{3} E \varepsilon_{0}^{4} \\
+\frac{1}{n}\left\|\boldsymbol{\alpha}^{*}\right\|_{1}^{4}\left|E\left(\varepsilon_{0}^{2}-E \varepsilon_{0}^{2}\right)^{2}-E^{*}\left(\varepsilon_{0}^{* 2}-E^{*} \varepsilon_{0}^{* 2}\right)^{2}\right| .
\end{gathered}
$$

This proves the lemma.

Proof of Theorem 4.1 : We first note that $S_{n}(p)$ and $S_{n}^{*}(p)$, cf. (2.8) and (4.1), can be written as

$$
S_{n}(p)=E\left\|A(p)\left(\tilde{\gamma}_{1}(p)-\gamma_{1}(p)\right)\right\|_{\Gamma(p)^{-1}}^{2}
$$

and

$$
S_{n}^{*}(p)=E^{*}\left\|A^{*}(p)\left(\tilde{\gamma}_{1}^{*}(p)-\gamma_{1}^{*}(p)\right)\right\|_{\Gamma^{*}(p)^{-1}}^{2},
$$

where the $p \times 2 p$-matrix $A(p)$ is defined as

$$
\left[\begin{array}{cccccccccc}
0 & 0 & \cdots & 0 & 0 & 1 & -a_{1}(p) & \cdots & -a_{p-1}(p) & -a_{p}(p) \\
0 & 0 & \cdots & 0 & 1 & -a_{1}(p) & -a_{2}(p) & \cdots & -a_{p}(p) & 0 \\
\vdots & & & \ddots & & & \ddots & & \vdots \\
0 & 1 & -a_{1}(p) & \cdots & & -a_{p}(p) & 0 & \cdots & 0 \\
1 & -a_{1}(p) & -a_{2}(p) & \ldots & -a_{p}(p) & 0 & \cdots & & 0
\end{array}\right]
$$

$\gamma_{1}(p):=\left(\gamma_{p}, \gamma_{p-1}, \ldots, \gamma_{1-p}\right)^{T} \in \mathbb{R}^{2 p}$ with $\gamma_{-h}=\gamma_{h}, A^{*}(p)$ is defined as $A(p)$ with $\tilde{a}_{\nu}(p)$ instead of $a_{\nu}(p)$ and $\tilde{\gamma}_{1}(p), \tilde{\gamma}_{1}^{*}(p), \gamma_{1}^{*}(p)$ are defined analogously to $\gamma_{1}(p)$.

Writing $\Sigma(p)=A(p)^{T} \Gamma(p)^{-1} A(p), \Sigma^{*}(p)=A^{*}(p)^{T} \Gamma^{*}(p)^{-1} A^{*}(p)$, we have

$$
\begin{aligned}
\left|S_{n}^{*}(p)-S_{n}(p)\right|= & \left|E^{*}\right|\left|\tilde{\gamma}_{1}^{*}(p)-\gamma_{1}^{*}(p)\right|_{\Sigma^{*}(p)}^{2}-E\left\|\tilde{\gamma}_{1}(p)-\gamma_{1}(p)\right\|_{\Sigma(p)}^{2} \mid \\
\leq & \left|E^{*}\right|\left|\tilde{\gamma}_{1}^{*}(p)-\gamma_{1}^{*}(p)\right|_{\Sigma^{*}(p)}^{2}-\left.E^{*}\left\|\tilde{\gamma}_{1}^{*}(p)-\gamma_{1}^{*}(p)\right\|\right|_{\Sigma(p)} ^{2} \mid \\
& +\left|E^{*}\left\|\tilde{\gamma}_{1}^{*}(p)-\gamma_{1}^{*}(p)\right\|_{\Sigma(p)}^{2}-E\left\|\tilde{\gamma}_{1}(p)-\gamma_{1}(p)\right\|_{\Sigma(p)}^{2}\right| .
\end{aligned}
$$

Bound the first summand through $\left\|\Sigma^{*}(p)-\Sigma(p)\right\| \cdot E^{*}\left\|\tilde{\gamma}_{1}^{*}(p)-\gamma_{1}^{*}(p)\right\|_{2}^{2}$. Because of Lemma 7.1 it suffices to show that

$$
\begin{aligned}
\max _{p}\left\|\Sigma^{*}(p)-\Sigma(p)\right\| & =\max _{p}\left\|A^{*}(p)^{T} \Gamma^{*}(p)^{-1} A^{*}(p)-A(p)^{T} \Gamma(p)^{-1} A(p)\right\| \\
& =o_{P}(1) .
\end{aligned}
$$

As $\|\Gamma(p)\|$ and $\left\|\Gamma(p)^{-1}\right\|$ are uniformly bounded in $p \in \mathbb{N}$, the last equation will follow from

$$
\max _{p}\left\|A^{*}(p)-A(p)\right\|, \max _{p}\left\|\Gamma^{*}(p)^{-1}-\Gamma(p)^{-1}\right\|=o_{P}(1), \max _{p}\|A(p)\|=O(1) .
$$


In view of the matrix inequalities $\|B\| \leq\|B\|_{2}:=\left(\sum\left|b_{i j}\right|^{2}\right)^{\frac{1}{2}}$ and $\left\|B^{-1}-C^{-1}\right\| \leq$ $\frac{\left\|B^{-1}\right\|^{2}\|B-C\|}{1-\left\|B^{-1}|\|\mid B-C\|\right.}$ if $\left\|B^{-1}\right\|\|B-C\|<1$, we obtain from Lemma 7.1

$$
\begin{gathered}
\max _{p}\|\tilde{\Gamma}(p)-\Gamma(p)\|=o_{P}(1), \\
\max _{p}\left\|\tilde{\Gamma}(p)^{-1}-\Gamma(p)^{-1}\right\|=o_{P}(1) .
\end{gathered}
$$

As $\Gamma^{*}(p)=\frac{\gamma_{0}^{*}}{\tilde{\gamma}_{0}} \tilde{\Gamma}(p)$ and $\frac{\gamma_{0}^{*}}{\tilde{\gamma}_{0}} \rightarrow 1$ in probability, (7.6) and $(7.7)$ hold with $\tilde{\Gamma}(p)$ replaced by $\Gamma(p)^{*}$.

Now, for any $x \in \mathbb{R}^{2 p}$ the vector $A(p) x$ obviously consists of certain entries of the convolution $\mathbf{j}(a(p)) * x^{-}$where $\mathbf{j}(a(p)), x$ are embedded in $\mathbb{R}^{\mathbb{Z}},\left(x^{-}\right)_{h}=x_{-h}$ and convolution takes place over $\mathbb{Z}$. From the convolution inequality $\|\mathbf{b} * \mathbf{c}\|_{2} \leq\|\mathbf{b}\|_{2}\|\mathbf{c}\|_{1}$ we conclude

$$
\|A(p)\|=\sup _{\|x\|_{2}=1}\|A(p) x\|_{2} \leq 1+\|a(p)\|_{1},
$$

where the latter is bounded uniformly in $p \in \mathbb{N}$ according to Theorem 2.2 of Baxter (1962). As by Lemma 7.1 and (7.7)

$$
\begin{aligned}
\max _{1 \leq p \leq p(n)}\|\tilde{a}(p)-a(p)\|_{2} & =\max _{p}\left\|\tilde{\Gamma}(p)^{-1} \tilde{\gamma}(p)-\Gamma(p) \gamma(p)\right\|_{2} \\
& =O_{P}\left(\sqrt{\frac{p(n)^{3}}{n}}\right)=o_{P}\left(\frac{1}{\sqrt{p(n)}}\right),
\end{aligned}
$$

we get in the same manner as in (7.8)

$$
\max _{p}\left\|A^{*}(p)-A(p)\right\| \leq \max _{p}\|\tilde{a}(p)-a(p)\|_{1}=o_{P}(1) .
$$

For the second summand we have

$$
\begin{aligned}
& \left|E^{*}\right|\left|\tilde{\gamma}_{1}^{*}(p)-\gamma_{1}^{*}(p)\left\|_{\Sigma(p)}^{2}-E\right\| \tilde{\gamma}_{1}(p)-\gamma_{1}(p)\right|_{\Sigma(p)}^{2} \mid \\
\leq & \sum_{h, k=1}^{p}\left|\Sigma(p)_{h k}\right|\left|\operatorname{Cov}^{*}\left(\tilde{\gamma}_{h}^{*}, \tilde{\gamma}_{k}^{*}\right)-\operatorname{Cov}\left(\tilde{\gamma}_{h}, \tilde{\gamma}_{k}\right)+\frac{h k}{n^{2}}\left(\tilde{\gamma}_{h} \tilde{\gamma}_{k}-\gamma_{h} \gamma_{k}\right)\right| .
\end{aligned}
$$

Now, from Shibata (1980), p. 151, there is a constant $C$ such that $\sum_{h, k=1}^{p}\left|\Gamma(p)_{h, k}^{-1}\right| \leq C p$ for all $p \in \mathbb{N}$. Hence we have $\sum_{h, k=1}^{p}\left|\Sigma(p)_{h k}\right| \leq C p\left(1+\|a(p)\|_{1}\right)^{2}$, and by Lemma 7.1 and the uniform boundedness of $\|a(p)\|_{1}$,

$$
\max _{p} \frac{n}{p}\left|E^{*}\left\|\tilde{\gamma}_{1}^{*}(p)-\gamma_{1}^{*}(p)\right\|_{\Sigma(p)}^{2}-E\left\|\tilde{\gamma}_{1}(p)-\gamma_{1}(p)\right\|_{\Sigma(p)}^{2}\right|=o_{P}(1)
$$

This concludes the proof of Theorem 4.1. 
Proof of Theorem 5.1 : Because of Theorem 4.1 it suffices to consider

$$
\max _{1 \leq p \leq p(n)} \frac{\left|S_{n}(p)-\frac{p}{n} \sigma^{2}\right|}{\frac{p}{n}+\frac{\sigma^{2}(p)-\sigma^{2}}{\sigma^{2}}} .
$$

Using convention (5.1), (5.2) we obtain

$$
S_{n}(p)=E\|\tilde{\gamma}(p)-\tilde{\Gamma}(p) a(p)\|_{\Gamma(p)^{-1}}^{2}=E\left\|\frac{1}{n} \sum_{t} \varepsilon_{t}(a(p)) X_{t-1}(p)\right\|_{\Gamma(p)^{-1}}^{2}
$$

where $\varepsilon_{t}(a(p))=X_{t}-a(p)^{T} X_{t-1}(p)$. Now, since

$$
\begin{aligned}
E\left\|\frac{1}{n} \sum_{t} \varepsilon_{t} X_{t-1}(p)\right\|_{\Gamma(p)^{-1}}^{2} & =\frac{1}{n^{2}} \sum_{t} E \varepsilon_{t}^{2} E\left(X_{t-1}(p)^{T} \Gamma(p)^{-1} X_{t-1}(p)\right) \\
& =\frac{n-p(n)}{n} \frac{p}{n} \sigma^{2}
\end{aligned}
$$

we have to show

$$
\max _{p} \frac{\left|E\left\|\frac{1}{n} \sum_{t} \varepsilon_{t}(a(p)) X_{t-1}(p)\right\|_{\Gamma(p)-1}^{2}-E\left\|\frac{1}{n} \sum_{t} \varepsilon_{t} X_{t-1}(p)\right\|_{\Gamma(p)-1}^{2}\right|}{\frac{p}{n}+\frac{\sigma^{2}(p)-\sigma^{2}}{\sigma^{2}}}=o_{P}(1) .
$$

To this end consider

$$
\begin{aligned}
& \left|E\left\|\frac{1}{n} \sum_{t} \varepsilon_{t}(a(p)) X_{t-1}(p)\right\|_{\Gamma(p)^{-1}}^{2}-E \| \frac{1}{n} \sum_{t} \varepsilon_{t} X_{t-1}(p)\right|_{\Gamma(p)^{-1}}^{2} \mid \\
& \leq E\left\|\frac{1}{n} \sum_{t}\left(\varepsilon_{t}(a(p))-\varepsilon_{t}\right) X_{t-1}(p)\right\|_{\Gamma(p)^{-1}}^{2} \\
& +2 \sqrt{E\left\|\frac{1}{n} \sum_{t} \varepsilon_{t} X_{t-1}(p)\right\|_{\Gamma(p)^{-1}}^{2}} \sqrt{E\left\|\frac{1}{n} \sum_{t}\left(\varepsilon_{t}(a(p))-\varepsilon_{t}\right) X_{t-1}(p)\right\|_{\Gamma(p)^{-1}}^{2}} .
\end{aligned}
$$

Because of (7.12), we may restrict our attention to

$$
\max _{p} \frac{E\left\|\frac{1}{n} \sum_{t}\left(\varepsilon_{t}(a(p))-\varepsilon_{t}\right) X_{t-1}(p)\right\|_{\Gamma(p)-1}^{2}}{\frac{p}{n}+\frac{\sigma^{2}(p)-\sigma^{2}}{\sigma^{2}}} .
$$

Now, $\|\Gamma(p)\|$ and $\left\|\Gamma(p)^{-1}\right\|$ are uniformly bounded in $p \in I N, \sigma^{2}(p)-\sigma^{2}=\|\mathbf{a}-a(p)\|_{\Gamma}^{2}$ and

$$
\sqrt{E\left\|\frac{1}{n} \sum_{t}\left(\varepsilon_{t}(a(p))-\varepsilon_{t}\right) X_{t-1}(p)\right\|_{2}^{2}} \leq \sum_{h=1}^{p} \sqrt{E\left[\frac{1}{n} \sum_{t}\left(\varepsilon_{t}(a(p))-\varepsilon_{t}\right) X_{t-h}\right]^{2}} .
$$

Therefore it suffices to show

$$
E\left[\frac{1}{n} \sum_{t}\left(\varepsilon_{t}(a(p))-\varepsilon_{t}\right) X_{t-h}\right]^{2} \leq \frac{C}{n}\|\mathbf{a}-a(p)\|_{2}^{2}, \quad h=1, \ldots, p,
$$


where $C>0$ is a constant independent of $p$. For this purpose, we fix $p$ and write $Z_{t}:=$ $\varepsilon_{t}(a(p))-\varepsilon_{t}$. Then $Z_{t}=\sum_{\ell=0}^{\infty} \beta_{\ell} \varepsilon_{t-\ell}$ where $\boldsymbol{\beta}=(\mathbf{j}(a(p))-\mathbf{j}(\mathbf{a})) * \boldsymbol{\alpha}$ and $\boldsymbol{\alpha}=\mathbf{a}^{-1}$. With these notations, (7.13) becomes

$$
\frac{1}{n^{2}} \sum_{s, t} E Z_{s} X_{s-h} Z_{t} X_{t-h} \leq \frac{C}{n}\|\mathbf{a}-a(p)\|_{2}^{2}, \quad h=1, \ldots, p .
$$

Using the $\mathrm{MA}(\infty)$-representations of the processes $\left(X_{t}\right),\left(Z_{t}\right)$ and the orthogonality $E Z_{t} X_{t-h}=0, h=1, \ldots, p$, it is easy to see that for $s \leq t$

$$
\begin{aligned}
E Z_{s} X_{s-h} Z_{t} X_{t-h}= & E Z_{s} Z_{t} E X_{s-h} X_{t-h}+E Z_{s} X_{t-h} E Z_{t} X_{s-h} \\
& +\sum_{\ell=0}^{\infty} \beta_{\ell+h} \alpha_{\ell} \beta_{\ell+t-s+h} \alpha_{\ell+t-s}\left(E \varepsilon_{0}^{4}-3 \sigma^{4}\right) .
\end{aligned}
$$

Setting $\alpha_{\ell}, \beta_{\ell}=0$ if $\ell<0$ we note that for any $k \in \mathbb{Z}$

$$
E Z_{0} X_{k}=\sum_{\ell=-\infty}^{\infty} \beta_{\ell} \alpha_{\ell+k}=\sum_{\ell=-\infty}^{\infty} \beta_{\ell} \alpha_{-(-\ell-k)}=\left(\boldsymbol{\beta} * \boldsymbol{\alpha}^{-}\right)_{-k}
$$

where $\boldsymbol{\alpha}^{-}=\left(\alpha_{\ell}: \ell \in \mathbb{Z}\right)$ and convolution takes place over $\mathbb{Z}$. Similar expressions may be derived for the autocovariances $E Z_{0} Z_{k}$ and $E X_{0} X_{k}$. Summation of the first term on the right side of (7.15) over $t \geq s$ gives

$$
\begin{aligned}
\sum_{t \geq s}\left|E Z_{s} Z_{t} E X_{s-h} X_{t-h}\right| & =\sum_{t \geq 0}\left|\left(\boldsymbol{\beta} * \boldsymbol{\beta}^{-}\right)_{t}\left(\boldsymbol{\alpha} * \boldsymbol{\alpha}^{-}\right)_{t}\right| \\
& \leq\left\|\boldsymbol{\beta} * \boldsymbol{\beta}^{-}\right\|_{2}\left\|\boldsymbol{\alpha} * \boldsymbol{\alpha}^{-}\right\|_{2} \\
& \leq\|\mathbf{a}-a(p)\|_{2}^{2}\|\boldsymbol{\alpha}\|_{1}^{4}
\end{aligned}
$$

by repeated use of the convolution inequality $\|\mathbf{b} * \mathbf{c}\|_{2} \leq\|\mathbf{b}\|_{2}\|\mathbf{c}\|_{1}$ and $\left\|\mathbf{b}^{-}\right\|_{1}=\|\mathbf{b}\|_{1}$. Summation of the other two terms on the right side of (7.15) leads to similar expressions, so we may conclude (7.14).

\section{References}

Akaike, H. (1973b). Information theory and an extension of the maximum likelihood principle. Second International Symposium on Information Theory (B.N. Petrov and F. Csaki, eds.), Akademia Kiado, Budapest, 267-281.

Akaike, H. (1974). A new look at the statistical model identification. IEEE Trans. Automatic Control, AC 19, 716-723.

Baxter, G. (1962). An asymptotic result for the finite predictor. Math. Scand. 10, 137-144.

Behrens, J. (1990). Robust order selection for autoregressive processes. Dissertation. Universität Kaiserslautern.

Brockwell, P.J. and Davis, R.A. (1991). Time Series: Theory and Methods (Second 
Edition). Springer-Verlag, New York.

Bühlmann, P. (1997). Sieve bootstrap for time series. Bernoulli 3, 123-148.

Deistler, M. and Hannan, E.J. (1988). The Statistical Theory of Linear Systems. John Wiley, New York.

Kreiss, J.-P. (1988). Asymptotic statistical inference for a class of stochastic processes. Habilitationsschrift. Univ. of Hamburg.

Kreiss, J.-P. (1997). Asymptotical properties of residual bootstrap for autoregressions. J. Time Ser. Anal. (submitted).

Martin, R.D. (1983). The Cramér-Rao bound and robust M-estimates for autoregressions. Biometrika 69, 437-442.

Moser, M. (1997). Bootstrap-Ordnungswahl und M-Schätzung in linearer Autoregression. Dissertation. TU Braunschweig.

Pedersen, G.K. (1995). Analysis Now (Second Edition). Springer-Verlag, New York. Shibata, R. (1980). Asymptotically efficient selection of the order of the the model for estimating parameters of a linear process. Ann. Statist. 8, 147-164.

Jürgen Franke

Fachbereich Mathematik

Universität Kaiserslautern

Erwin-Schrödinger-Strasse

67663 Kaiserslautern

Germany
Jens-Peter Kreiss

Institut für Mathematische Stochastik

Technische Universität Braunschweig

Pockelstrasse 14

38106 Braunschweig

Germany

Martin Moser

Institut für Angewandte Mathematik

Universität Heidelberg

Im Neuenheimer Feld 294

69120 Heidelberg

Germany 